\title{
Nanoscale
}

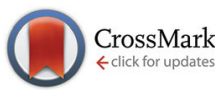

Cite this: Nanoscale, 2015, 7, 8294

Received 12th March 2015 Accepted 31st March 2015

DOI: $10.1039 / \mathrm{c} 5 \mathrm{nr} 01592 \mathrm{~h}$

www.rsc.org/nanoscale

\section{Collective excitation of plasmonic hot-spots for enhanced hot charge carrier transfer in metal/semiconductor contacts $\uparrow$}

\author{
Adrien Piot, ${ }^{\mathrm{a}, \mathrm{b}}$ Stuart K. Earl, ${ }^{\mathrm{c}}$ Charlene Ng, ${ }^{\mathrm{b}, \mathrm{d}}$ Svetlana Dligatch, ${ }^{\mathrm{e}}$ Ann Roberts, ${ }^{\mathrm{c}}$ \\ Timothy J. Davis ${ }^{b, d}$ and Daniel E. Gómez ${ }^{\star b, d}$
}

\begin{abstract}
We show how a combination of near- and far-field coupling of the localised surface plasmon resonances in aluminium nanoparticles deposited on $\mathrm{TiO}_{2}$ films greatly enhances the visible light photocatalytic activity of the semiconductor material. We demonstrate two orders of magnitude enhancement in the rate of decomposition of methylene blue under visible light illumination when the surface of $\mathrm{TiO}_{2}$ films is decorated with gratings of Al nanoparticle dimers.
\end{abstract}

Photocatalysis with semiconductor materials has great potential for the direct conversion of solar to chemical energy. A photocatalyst material after absorbing light energy, creates highly energetic charge carriers that can drive useful chemical transformations, including production of fuels by solar energy (e.g. hydrogen from water splitting ${ }^{1-5}$ and hydrocarbons from $\mathrm{CO}_{2}$ (ref. 6)), the production of high-value fine chemicals (e.g. ethylene oxide ${ }^{7}$ ) and the elimination of hazardous contaminants from air and water (e.g. nitrous oxides, etc.). Conventional photocatalytic processes are however not very efficient. ${ }^{8}$ Suitable semiconductor materials for photocatalysis (i) only absorb light in the UV region of the solar spectrum (less than $10 \%$ of the total available solar energy) (ii) exhibit fast recombination of the photo-excited charge carriers which additionally (iii) have small diffusion lengths, factors which add up to low photocatalytic efficiencies.

Plasmonic enhanced photocatalysis ${ }^{9-14}$ has resurfaced as a very effective approach for increasing the efficiency of photocatalytic processes. In this approach, nanoparticles of coinage

\footnotetext{
${ }^{a}$ Institut National des Sciences Appliquées de Toulouse, 135 Avenue de Rangueil, 31400 Toulouse, France

${ }^{b}$ Melbourne Centre for Nanofabrication, Australian National Fabrication Facility, Clayton, VIC 3168, Australia

${ }^{c}$ School of Physics, The University of Melbourne, Parkville, VIC 3010, Australia ${ }^{d}$ CSIRO, Manufacturing Flagship, Private Bag 33, Clayton, VIC 3168, Australia. E-mail:daniel.gomez@csiro.au

${ }^{e}$ CSIRO, Manufacturing Flagship, PO Box 218, Lindfield, NSW 2070, Australia $\dagger$ Electronic supplementary information (ESI) available: Detailed information on estimates of hot-electron injection efficiencies, electrodynamic simulations, sample preparation, spectroscopic and structural characterization and photocatalytic experiments. See DOI: 10.1039/c5nr01592h
}

metals (predominantly $\mathrm{Ag}$ and $\mathrm{Au}$ ) are placed either in direct contact or in the vicinity of the photocatalytic material, leading to increased generation of chemically useful electronhole pairs as a result of several surface plasmon related mechanisms that mitigate some of the intrinsic shortcomings listed above.

One such mechanism involves the transfer of hot-electrons from metal nanoparticles into the photocatalytic material, in an analogous manner to dye sensitization in solar cells. ${ }^{15-17}$ Absorption of light by metal nanoparticles, through photoexcitation of localised surface plasmon resonances (LSPRs), can result (after non-radiative relaxation) in the formation of hot-electrons: a transient population of electrons with energies between the vacuum and the Fermi level of the metal. ${ }^{14}$ When the metal is in direct contact with the semiconductor material, a Schottky energy barrier $\phi_{\mathrm{SB}}$ occurs (see Fig. 1) and the efficiency of hot-electron transfer is proportional to the fraction of the hot-electron population which has enough energy to overcome this barrier. ${ }^{18}$ Given that in general $\phi_{\mathrm{SB}}$ is much

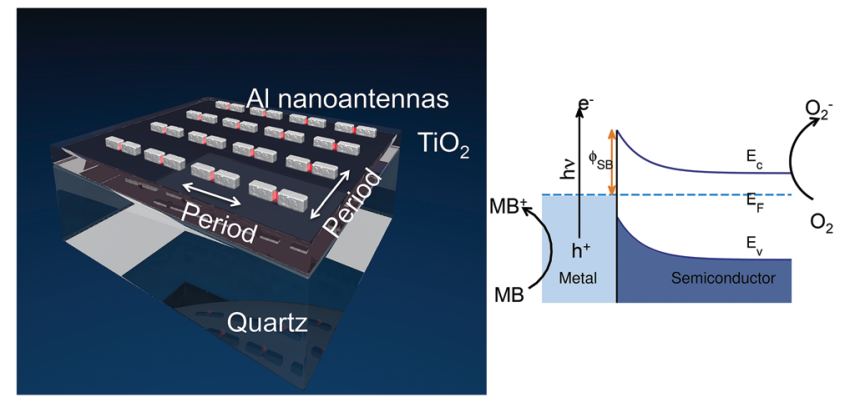

Fig. 1 Aluminium gap nanoantennas are deposited on top of $190 \mathrm{~nm}$ films of $\mathrm{TiO}_{2}$. The antennas are periodically arranged on the substrate. The periodicity of the array is optimised to achieve a collective in-phase dipolar coupling on the nanoantennas. The diagram on the right illustrates the idealised energy band alignment at the metal/semiconductor interface. Non-radiative plasmon relaxation produces a hot-electron that is transferred above the Schottky barrier $\phi_{\mathrm{SB}}$ to $\mathrm{TiO}_{2}$. The charge separated state initiates charge transfer processes in solution. 
smaller than the band-gap of the semiconducting material, the generation of chemically useful ${ }^{16}$ charge carriers via this mechanism extends the response of the combined photocatalytic system to the visible and near-infrared regions of the spectrum. ${ }^{19}$

According to White and Catchpole, ${ }^{18}$ the metal-to-semiconductor hot-electron photo-current $j_{\mathrm{sc}}$ can be estimated with the following equation:

$$
j_{\mathrm{sc}}=e \int S(\lambda) \sigma_{\mathrm{abs}}(\lambda) \eta\left(\lambda, \phi_{\mathrm{SB}}\right) \mathrm{d} \lambda
$$

where $e$ is the electron's charge, $S(\lambda)$ is the incident photon flux [number of photons/( $\left.\left(\mathrm{nm} \mu \mathrm{m}^{2}\right)\right], \sigma_{\mathrm{abs}}(\lambda)$ is the absorption cross-section $\left(\mu \mathrm{m}^{2}\right)$ of the metal nanoparticles and $\eta\left(\lambda, \phi_{\mathrm{SB}}\right)$ is the probability of hot-electron emission from the metal nanoparticles into the semiconductor material.

The Schottky barrier between $\mathrm{Au}$ and $\mathrm{TiO}_{2}$ is in the order of 0.9 to $1.0 \mathrm{eV}^{20}$ which implies that the fraction of hot-electrons with sufficient energy to cross the barrier $\eta\left(\lambda, \phi_{\mathrm{SB}}\right)$ is about $20 \%$ to $40 \%{ }^{21}$ at the wavelengths of the LSPRs ${ }^{18}$ (see the ESI $\dagger$ ). In spite of this limitation, several reports exist where Au nanoparticles have greatly enhanced the photocatalytic activity (and photocurrents) of $\mathrm{TiO}_{2}{ }^{22}$ The fraction $\eta\left(\lambda, \phi_{\mathrm{SB}}\right)$ of hot-electrons could be increased to $>70 \%$ by using aluminium, an abundant and relatively inexpensive metal that has a more positive work function and consequently a smaller associated metal-semiconductor Schottky barrier (see detailed estimate in the ESI $\dagger$ ).

According to eqn (1), the second parameter that affects the hot-electron photocurrent is $\sigma_{\mathrm{abs}}(\lambda)$, the optical absorption cross section of the metal nanoparticles which can be controlled by the shape and geometrical arrangement of the metal particles. Al nanoparticles typically exhibit localised surface plasmon resonances in the UV region ${ }^{23-26}$ but when placed in direct contact with high refractive index materials, such as $\mathrm{TiO}_{2}$, these LSPRs can be shifted to the visible light. ${ }^{27}$

In this communication, we have demonstrated how diffractive coupling in $\mathrm{Al}$ nanoparticles leads to significant enhancements in the photocatalytic activity of $\mathrm{TiO}_{2}$ using visible light. Our approach exploits plasmonic coupling effects at two length scales to maximise visible light absorption by the plasmonic array. The concept is illustrated in Fig. 1. The unit cell of the plasmonic arrays consists of Al nanorod dimers with tipto-tip separation distances of about $20 \mathrm{~nm}$. At these deep subwavelength distances, near-field interactions lead to plasmonic hybridization $^{28}$ resulting in local electromagnetic field densities that can be many orders of magnitude higher than the incident field (i.e. plasmonic hot-spots), ${ }^{29}$ an effect that has recently been employed for achieving one order of magnitude enhancement in surface enhanced infra-red spectroscopy. ${ }^{30}$ These $\mathrm{Al}$ dimers are periodically arranged on the surface of the thin $(190 \mathrm{~nm})$ films of $\mathrm{TiO}_{2}$ with a period that results in a collective excitation of all the dimers in the array and consequently in the absorption of light that far exceeds that of the non-interacting Al dimers. ${ }^{31}$

Light absorbed by these strongly coupled arrays results, after surface plasmon non-radiative relaxation, in hot charge carrier transfer from the $\mathrm{Al}$ dimers to the semiconductor substrate, an efficient process for $\mathrm{Al} / \mathrm{TiO}_{2}$ in the visible region of the spectrum. $\$$ This charge transfer initiates redox reactions at the metal and semiconductor interfaces.

We arbitrarily choose the geometry of the unit cell of the arrays of Fig. 1 for it to exhibit particle plasmon resonances in the visible region of the spectrum. With a fixed geometry for the unit cell, the next step in the design consists of optimising the value of the periodicity of the lattice required to achieve inphase diffractive dipole-dipole interactions: ${ }^{32-37}$ for a single $\mathrm{Al}$ dimer, the driving electromagnetic field is simply given by the incident field $\overrightarrow{\mathbf{E}}_{\text {inc }}$. For a periodic array, the local field $\overrightarrow{\mathbf{E}}_{\text {loc }}$ that excites each nanoparticle is a superposition of the incident field and the field created by the other nanoparticles in the array:

$$
\overrightarrow{\mathbf{E}}_{\mathrm{loc}}=\overrightarrow{\mathbf{E}}_{\mathrm{inc}}+\sum_{p} \overrightarrow{\mathbf{E}}_{p}
$$

where the summation is implicitly defined to exclude selfinteraction terms. The geometry of the plasmonic array dictates the relative contribution of the second term to the total local electric field, which can result in suppressed extinction ${ }^{33}$ or extremely large local electric fields. ${ }^{35}$

In Fig. 2(A) we show how the calculated absorption spectra change with the periodicity of the array where in addition to spectral red-shifts with increasing period, changes in the mag-
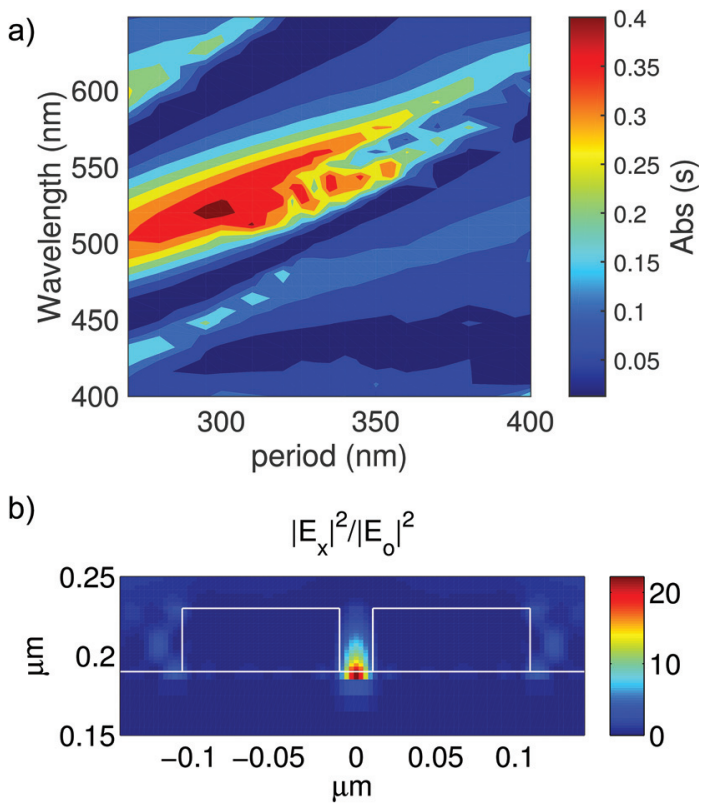

Fig. 2 (A) Calculated absorption spectra of Al dimer arrays for different periods for s polarization. The width of each nanorod was $80 \mathrm{~nm}$, length $100 \mathrm{~nm}$, height $40 \mathrm{~nm}$ and the face-to-face distance was fixed at $20 \mathrm{~nm}$. The metal particles were assumed to be deposited on top of $190 \mathrm{~nm}$ $\mathrm{TiO}_{2}$ thin films. (B) Calculated spatial distribution of the $x$-component of the electric field at the $520 \mathrm{~nm}$ resonance. It is clear that a plasmonic hot-spot occurs at the gap of the nanorods and furthermore most of this electric field is confined to the $\mathrm{TiO}_{2}$ space. 
nitude of the absorption are also evident. The dominant absorption band reaches a maximum for an array period of $300 \mathrm{~nm}$; this enhanced absorption originates from the inphase collective oscillation of the LSPRs of the Al dimers. These bands occur at wavelengths $\lambda$ where the diffractive order $(i, j)$ of the periodic array transitions from evanescent to radiative, ${ }^{31}$ a condition approximately given by: ${ }^{36}$

$$
\lambda \approx \frac{p}{\sqrt{i^{2}+j^{2}}} n_{\mathrm{TiO}_{2}},
$$

which for a period of $p=300 \mathrm{~nm}$, a refractive index $n_{\mathrm{TiO}_{2}} \sim$ 2.45 and for the diffraction order for which $i=j=1$ occurs at wavelengths around $\lambda \sim 520 \mathrm{~nm}$, a wavelength where the $\mathrm{Al}$ nanodimers focus electromagnetic energy very strongly at the inter-particle gap. To illustrate this point, we have shown in Fig. 2(B) a map of the $x$-component of the magnitude (squared) of the electric field. This represents the contribution of field enhancement due to the excitation of the longitudinal hybrid plasmon mode of the nanoparticle dimer. Due to the asymmetric dielectric environment, these hot-spots are more concentrated at the $\mathrm{TiO}_{2}$ region.

To realise these systems that exhibit collective excitation of plasmonic hot-spots, we fabricated arrays of $\mathrm{Al}$ nanorod dimers using top-down electron beam lithography on glass slides coated with $190 \mathrm{~nm} \mathrm{TiO}_{2}$ thin films. $\S$ This top-down nanofabrication approach results in naked surfaces (i.e. free of ligands) which favour interfacial charge transfer. A scanning electron microscopy image of one such array is shown in Fig. 3(A). In Fig. 3(B) we show the spectrum of the extinction

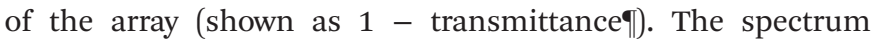
exhibits many spectral features but the dominant one occurs around $520 \mathrm{~nm}$ in close agreement with the estimate given by eqn (2) and the numerical simulations of Fig. 2.

The spectral band at $520 \mathrm{~nm}$ is very narrow and intense, which is characteristic of the excitation of a collective mode with suppressed radiative damping. ${ }^{35}$ The spectrum also has regions of very suppressed absorptions (at $\sim 490 \mathrm{~nm}$ and $\sim 600 \mathrm{~nm}$ ) which could be accounted for by considering the destructive interference between the waveguided modes at the $\mathrm{TiO}_{2}$ films and the particle plasmon resonances. ${ }^{33,38}$ At this $520 \mathrm{~nm}$ wavelength, light normally incident on the array excites a collective plasmonic oscillation which concentrates most of the electromagnetic energy on the hot spots located at the nanodimer gap [see Fig. 2(B)]. Non-radiative relaxation of this collective oscillation could result in the excitation of hot electrons on the Al nanodimers which could be transferred into the underlying $\mathrm{TiO}_{2}$ film.

As a test of this hypothesis, we measured the rate of degradation of methylene blue (MB) by following spectroscopically a decrease in the absorption band of $\mathrm{MB}$ at $665 \mathrm{~nm}$ upon illumination with spectrally filtered light from a $\mathrm{Hg}$ lamp (centred at $550 \mathrm{~nm}$ with a $40 \mathrm{~nm}$ band pass). MB decomposition by $\mathrm{TiO}_{2}$ is a convenient test reaction because it can be followed spectroscopically in the visible region of the spectrum and it is also a well understood reaction that is initiated by charge transfer a)

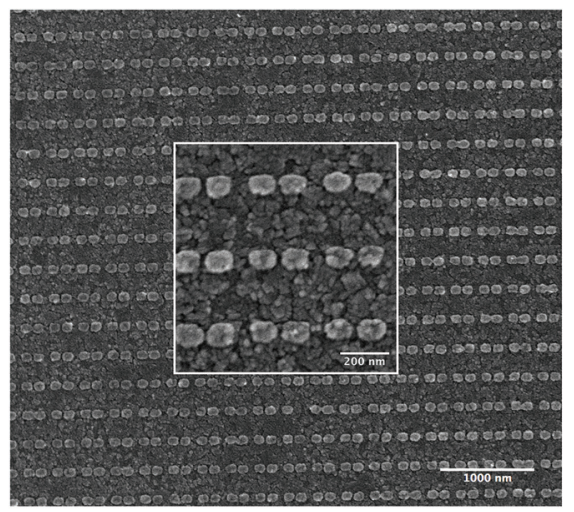

b)
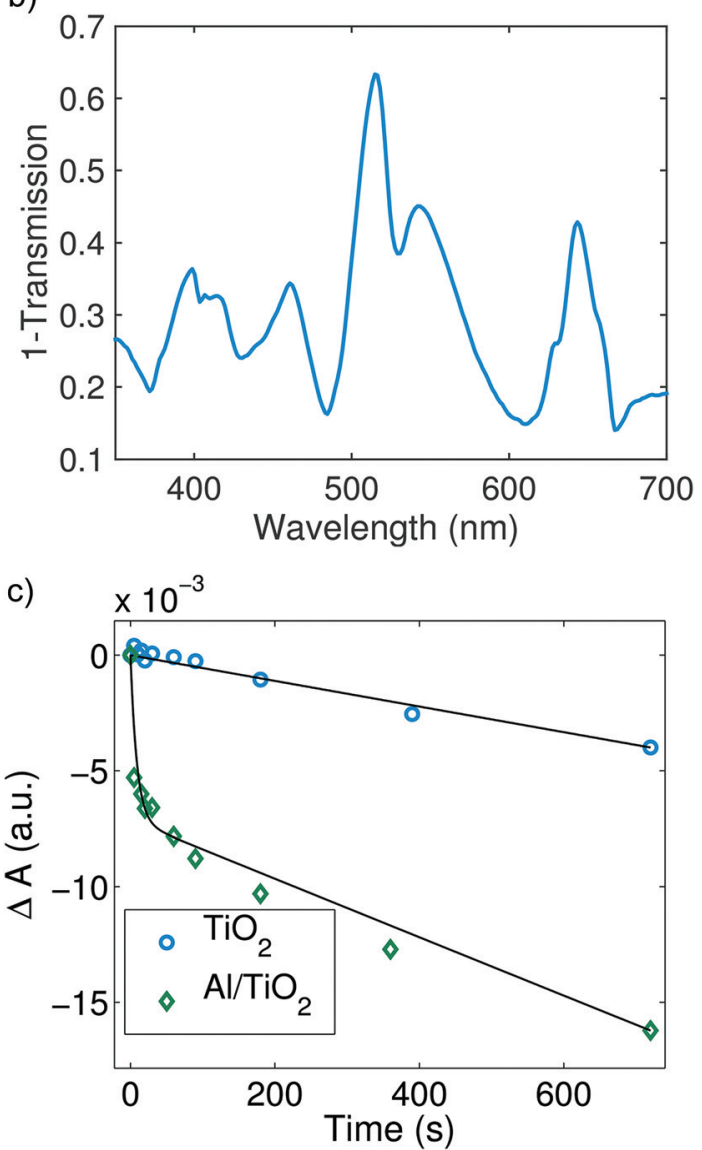

Fig. 3 (A) A scanning electron microscopy (SEM) image of a square array of Al nanodimers with a period of $300 \mathrm{~nm}$, shown at two different size scales. (B) Extinction spectra $(1-T)$ of the fabricated arrays measured with unpolarised light. (C) Change in absorbance of a $50 \mathrm{ppm}$ solution of methylene blue contained inside a microfluidic channel on top of a $3 \times 3 \mathrm{~mm}^{2}$ array of Al nanoantenna dimers with $540 \mathrm{~nm}$ illumination (bandwidth of $40 \mathrm{~nm}$ ).

processes as depicted in Fig. $1:^{39}$ the $\mathrm{Al}$ nanoparticles inject holes into $\mathrm{MB}$ molecules and the $\mathrm{TiO}_{2}$ film injects electrons into dissolved oxygen. The resulting charged species undergo a number of transformations that eventually lead to a net decomposition of MB into smaller molecules. ${ }^{39}$ 
The measured absorbance decay $(\Delta A)$ (Fig. 3(C)) is directly proportional to the change in concentration of $\mathrm{MB}$. For the bare $\mathrm{TiO}_{2}$ substrate, the observed $\Delta A$ time decay was accurately described by a first-order kinetic decay (single exponential) yielding a rate constant of $k=5.6 \times 10^{-6} \mathrm{~s}^{-1}$. In contrast, for the $\mathrm{Al}$ nanodimer array we found that the decay was bi-exponential with rates $k_{1}=1.13 \times 10^{-1} \mathrm{~s}^{-1}$ and $k_{2}=1.28 \times 10^{-5} \mathrm{~s}^{-1}$, which are $\sim 10^{4}$ and $\sim 2$ times higher than the one obtained for the bare $\mathrm{TiO}_{2}$ substrate. An average decay rate $\langle k\rangle=8.18 \times 10^{-4}$ $\mathrm{s}^{-1}$ can be estimated from the bi-exponential fit $\|$ which is about 150 times larger than the one observed for the bare $\mathrm{TiO}_{2}$ substrate (which exhibits a low rate due to its poor absorption of visible light). This result would seem to confirm our hypothesis and furthermore, it seems to imply that hole transfer from $\mathrm{Al}$ nanoparticles to $\mathrm{MB}$ molecules in solution [see Fig. 1] occurs in spite of the documented evidence that Al nanoparticles self-terminate with a 3-5 $\mathrm{nm}$ oxide layer when exposed to ambient air. $^{26}$

We have demonstrated how the periodic arrangement of metal nanoparticles on a photocatalytic substrate leads to strong collective plasmon resonances. These strong optical responses are translated into augmented rates of hot-electron generation and consequent enhanced redox reaction rates. Our proof-of-concept demonstration is not just limited to variations in the periodicity of deposited nanostructures; in contrast, lithographic nanofabrication enables unparalleled control over the geometry of the nanoparticles, which we anticipate to have as yet to be explored effects on the enhancement of photochemical processes.

This work was performed in part at the Melbourne Centre for Nanofabrication (MCN) in the Victorian Node of the Australian National Fabrication Facility (ANFF). A. P. was jointly supported by the MCN and CSIRO. C. N. was supported by an OCE Fellowship from CSIRO. D. E. G. acknowledges the ARC for support through a Future Fellowship (FT140100514) and F. Eftekhari for fruitful advise on EBL and L. Hyde for his help on ellipsometric measurements. D. E. G. and T. J. D. acknowledge the ANFF for the MCN Technology Fellowships.

\section{References}

$\ddagger$ See the ESI. $\dagger$

$\S$ Full details in the ESI. $\dagger$

- Details of measurement and data processing in the ESI. $\dagger$

$\|$ Details in the ESI. $\dagger$

1 H. M. Chen, C. K. Chen, C.-J. Chen, L.-C. Cheng, P. C. Wu, B. H. Cheng, Y. Z. Ho, M. L. Tseng, Y.-Y. Hsu, T.-S. Chan, J.-F. Lee, R.-S. Liu and D. P. Tsai, ACS Nano, 2012, 6, 73627372.

2 J. Lee, S. Mubeen, X. Ji, G. D. Stucky and M. Moskovits, Nano Lett., 2012, 12, 5014-5019.

3 D. B. Ingram and S. Linic, J. Am. Chem. Soc., 2011, 133, 5202-5205.

4 Z. Liu, W. Hou, P. Pavaskar, M. Aykol and S. B. Cronin, Nano Lett., 2011, 11, 1111-1116.
5 I. Thomann, B. A. Pinaud, Z. Chen, B. M. Clemens, T. F. Jaramillo and M. L. Brongersma, Nano Lett., 2011, 11, 3440-3446.

6 W. Hou, W. H. Hung, P. Pavaskar, A. Goeppert, M. Aykol and S. B. Cronin, ACS Catal., 2011, 1, 929-936.

7 P. Christopher, H. Xin and S. Linic, Nat. Chem., 2011, 3, 467-472.

8 M. Gratzel, Nature, 2001, 414, 338-344.

9 S. C. Warren and E. Thimsen, Energy Environ. Sci., 2012, 5, 5133-5146.

10 S. Linic, P. Christopher, H. Xin and A. Marimuthu, Acc. Chem. Res., 2013, 46, 1890.

11 X. Zhang, Y. L. Chen, R.-S. Liu and D. P. Tsai, Rep. Prog. Phys., 2013, 76, 046401.

12 M. Xiao, R. Jiang, F. Wang, C. Fang, J. Wang and J. C. Yu, J. Mater. Chem. A, 2013, 1, 5790-5805.

13 M. J. Kale, T. Avanesian and P. Christopher, ACS Catal., 2014, 4, 116-128.

14 C. Clavero, Nat. Photonics, 2014, 8, 95-103.

15 Y. Tian and T. Tatsuma, J. Am. Chem. Soc., 2005, 127, 76327637.

16 S. Linic, P. Christopher and D. B. Ingram, Nat. Mater., 2011, 10, 911-921.

17 A. Furube, L. Du, K. Hara, R. Katoh and M. Tachiya, J. Am. Chem. Soc., 2007, 129, 14852-14853.

18 T. P. White and K. R. Catchpole, Appl. Phys. Lett., 2012, 101, 073905.

19 Y. Nishijima, K. Ueno, Y. Kotake, K. Murakoshi, H. Inoue and H. Misawa, J. Phys. Chem. Lett., 2012, 3, 12481252.

20 Y. Nishijima, K. Ueno, Y. Yokota, K. Murakoshi and H. Misawa, J. Phys. Chem. Lett., 2010, 1, 2031-2036.

21 S. Mubeen, G. Hernandez-Sosa, D. Moses, J. Lee and M. Moskovits, Nano Lett., 2011, 11, 5548-5552.

22 Z. Bian, T. Tachikawa, P. Zhang, M. Fujitsuka and T. Majima, J. Am. Chem. Soc., 2014, 136, 458-465.

23 C. Langhammer, M. Schwind, B. Kasemo and I. Zorić, Nano Lett., 2008, 8, 1461-1471.

24 J. M. McMahon, G. C. Schatz and S. K. Gray, Phys. Chem. Chem. Phys., 2013, 15, 5415-5423.

25 J. M. Sanz, D. Ortiz, R. Alcaraz de la Osa, J. M. Saiz, F. González, A. S. Brown, M. Losurdo, H. O. Everitt and F. Moreno, J. Phys. Chem. C, 2013, 117, 1960619615.

26 M. W. Knight, N. S. King, L. Liu, H. O. Everitt, P. Nordlander and N. J. Halas, ACS Nano, 2014, 8, 834-840.

27 O. Lecarme, Q. Sun, K. Ueno and H. Misawa, ACS Photonics, 2014, 1, 538-546.

28 E. Prodan, C. Radloff, N. J. Halas and P. Nordlander, Science, 2003, 302, 419-422.

29 J. Aizpurua, G. W. Bryant, L. J. Richter, F. J. García de Abajo, B. K. Kelley and T. Mallouk, Phys. Rev. B: Condens. Matter, 2005, 71, 235420.

30 C. Huck, F. Neubrech, J. Vogt, A. Toma, D. Gerbert, J. Katzmann, T. Härtling and A. Pucci, ACS Nano, 2014, 8, 4908-4914. 
31 R. Adato, A. A. Yanik, J. J. Amsden, D. L. Kaplan, F. G. Omenetto, M. K. Hong, S. Erramilli and H. Altug, Proc. Natl. Acad. Sci. U. S. A., 2009, 106, 19227-19232.

32 B. Lamprecht, G. Schider, R. T. Lechner, H. Ditlbacher, J. R. Krenn, A. Leitner and F. R. Aussenegg, Phys. Rev. Lett., 2000, 84, 4721-4724.

33 S. Linden, J. Kuhl and H. Giessen, Phys. Rev. Lett., 2001, 86, 4688-4691.

34 C. L. Haynes, A. D. McFarland, L. L. Zhao, R. P. Van Duyne, G. C. Schatz, L. Gunnarsson, J. Prikulis, B. Kasemo and M. Kall, J. Phys. Chem. B, 2003, 107, 7337-7342.
35 B. Auguié and W. L. Barnes, Phys. Rev. Lett., 2008, 101, 143902.

36 V. G. Kravets, F. Schedin and A. N. Grigorenko, Phys. Rev. Lett., 2008, 101, 087403.

37 G. Vecchi, V. Giannini and J. Gómez Rivas, Phys. Rev. B: Condens. Matter, 2009, 80, 201401.

38 A. Christ, S. G. Tikhodeev, N. A. Gippius, J. Kuhl and H. Giessen, Phys. Rev. Lett., 2003, 91, 183901.

39 A. Houas, H. Lachheb, M. Ksibi, E. Elaloui, C. Guillard and J.-M. Herrmann, Appl. Catal., B, 2001, 31, 145157. 